\title{
ENERGIA UNDIMOTRIZ, ALTERNATIVA PARA LA PRODUCCIÓN DE ENERGÍA ELÉCTRICA EN LA PROVINCIA DE SANTA ELENA"
}

\author{
Rolando Calero Mendoza ${ }^{1}$, David Viteri Rivera ${ }^{1}$ \\ 1 Facultad de Ingeniería Industrial \\ Universidad Estatal Península De Santa Elena (UPSE) \\ Campus La Libertad, vía principal Santa Elena - La Libertad \\ La Libertad-Ecuador \\ rcalero@upse.edu.ec
}

\begin{abstract}
RESUMEN
La energía undimotriz, es la energía que permite obtener electricidad a partir de energía mecánica generada por el movimiento de las olas. Es uno de los tipos de energías renovables con más recientes estudios, y presenta enormes ventajas frente a otras energías renovables debido a que se tendría mayor facilidad para predecir condiciones geológicas óptimas que permitan la mayor eficiencia en sus procesos. Debido a que es más fácil llegar a predecir condiciones óptimas de oleaje, en comparación con la obtenida con los vientos para obtener energía eólica, donde su variabilidad es menor.En este trabajo se espera aportar y dilucidar con datosespecíficos sobre la morfología de la zonamarítima en la provincia de Santa Elena, tratando depredecir si las características propias del lugar son idóneas para producir electricidad en base a la implementación de energíaundimotriz, como fuente renovable no convencional utilizando las tecnologías actuales para este propósito.
\end{abstract}

Palabras claves: Energía renovable, Energía oceánica, Energía undimotriz, Olas, tecnologías actuales.

\begin{abstract}
Wave energyis the energythatallows the production ofelectricity frommechanical energy generatedby the movement ofthe waves.It isone of thetypes of renewable energywithrecentstudies, andhas hugeadvantages overother renewable energybecauseitcomesmore easilyto predictoptimalgeological conditionsthat allowgreater efficiencyin their processes.Because it iseasier to reachoptimumswellforecast, compared with that obtained withthe windsforwind energy, since its variabilityis less. This paperhopes to contributeandelucidatespecific facts aboutthe morphology ofthe sea areain the provinceof SantaElena, to predict whetherthe characteristicsof the siteare suitablefor electricity productionbased on theimplementation ofwave energysuch asnon-conventional renewablesource using the current technologies for this purpose.
\end{abstract}

Keywords: Renewable energy, oceanic energy, wave energy, waves, current technologies. 


\section{Introducción}

Los océanos son una de las fuentes de energías no convencionales más importantes que existen en nuestro planeta, debido a su magnitud y asu ilimitado alcance, aunquese han realizado pocos estudios que nospermitadesarrollar esta alternativa energética, actualmente existe en muchos países algunos logros al respecto en donde las características morfológicas son propicias para su utilización ${ }^{1-3}$. En el caso de Ecuador aún no cuenta con información suficiente que permita el desarrollo de este tipo de energía renovable para su posterior explotación.Se conoce que el oleaje que arriba a la costa del Ecuador tiene un promedio de olas de $2 \mathrm{~m}$, en profundidades de hasta $20 \mathrm{~m}$ y de $2.5 \mathrm{~m}$ en profundidades superiores, sin embargo estos valores son solo referenciales, dado que una vez que las olas ingresan a aguas costeras varían considerablemente por influencia del fondo, efectos de refracción, difracción entre otros.

La contaminación del medio ambiente es sin lugar a dudas uno de los principales factores que obliga a buscar mecanismos o métodos para la producción de energía eléctrica de una forma no agresiva para el medio ambiente, es decir la producción de energía sinemisión de gases, sólidos o líquidos contaminantes, lo que ocasiona aumento de la polución, alteración drástica del ecosistema y a la postreser causante del calentamiento global con sus consecuencias irremediables como aceleración del derretimiento de los casquetes polares, contaminación de la tierra por la lluvia acida, o la contaminación de nuestro aire por causa de la emisión de gases de efecto invernadero, en definitiva cambios violentos del clima a nivel mundial. ${ }^{4}$

Existe actualmente una falta de información sobre los recursos naturales con potencial energético para el Ecuador. Con excepción de la energía hídrica, las demás energías no cuentan con información suficiente para determinar el verdadero potencial energético de nuestro país.

Las olas de los mares y océanos son un derivado terciario de la energía solar, pues la radiación solar incide sobre la superficie de la Tierra y provoca un calentamiento desigual de la misma, produciendo en la atmósfera zonas con distinta presión, lo que produce desplazamientos de aire de uno a otro lugar, es decir generando vientos.

Y son estos vientos, los que al desplazarse sobre la superficie del mar llevan a cabo el rozamiento de las moléculas de aire con el agua, transfiriendo a esta parte de su energía y generando las olas, estas actúan como un acumulador de energía pues la almacenan y la transportan de un lugar a otro sin apenas perdidas, lo que da lugar a que la energía de las olas que se producen en cualquier parte del océano acabe en las costas.

Tomando en consideración la ley de régimen del sector eléctrico artículo 5, literal j que dice: "Desarrollar la electrificación en el sector rural"; y, literal k que dice: "Fomentar el desarrollo y uso de los recursos energéticos no convencionales a través de los organismos públicos, las universidades y las instituciones privadas"; y el artículo 63 que dice que "el estado fomentara el desarrollo y uso de los recursos energéticos no convencionales a través de los organismos públicos, la banca de desarrollo, las universidades y las instituciones privadas". 5

EL CONELEC asignará con prioridad fondos del FERUM a proyectos de electrificación rural a base de recursos energéticos no convencionales tales como energía solar, eólica, geotérmica, biomasa y otras de similares características”, artículo 64 "El Consejo Nacional de Electrificación dictará las normas aplicables para el despacho de la electricidad producidas con energías no convencionales tendiendo a su aprovechamiento y prioridad ".

En este artículose hace una revisión sobre dispositivos flotadores, específicamente la boya de energía (Powerbuoy) del OPT (OceanPower Technologies) ${ }^{6}$ proponiendo a su vez un modelo de dispositivoque podría ser implementado frente a la costa de la provincia de Santa Elena para la producción de electricidad.

Como objetivo se tiene el determinar la factibilidad de aplicar la energía undimotriz, para la obtención de electricidad para poder implementarla en la zona costeraecuatoriana, específicamente en algunas zonas que cubren la región costera en la provincia de Santa Elena, estudiando su viabilidad como una solución para la cobertura de la demanda nacional del sistema interconectado y de zonas aisladas.

El área de cobertura es laregión de la plataforma continental, A una distancia de la costa a1.5 km aproximadamente con olas de una altura variable de entre 2 y 2,5 m, en zonas con profundidades entre 20 y 100 metros. 


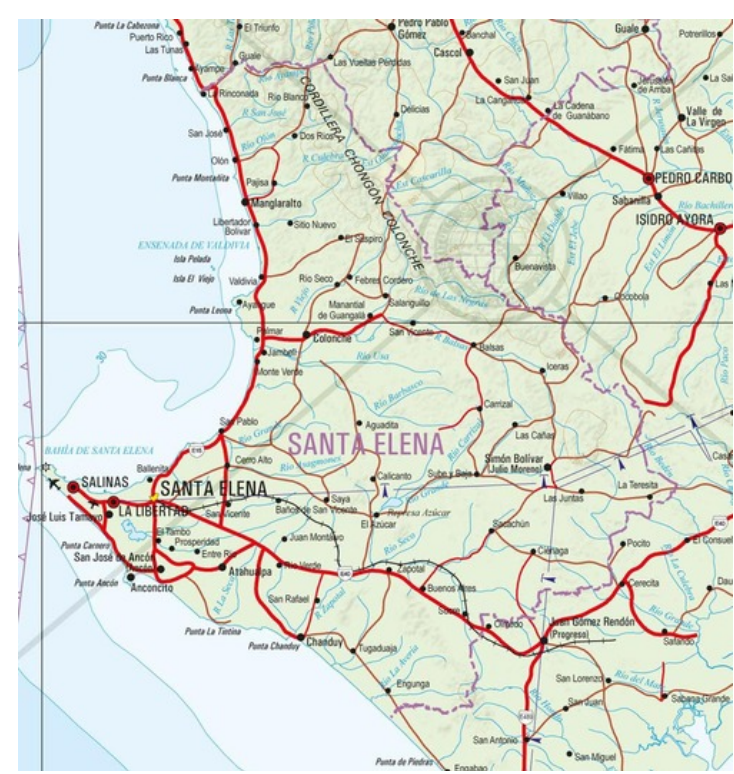

Figura 1.Mapa de la provincia de Santa Elena(Fuente: Instituto Geográfico Militar)

Morfología del suelo oceánico: el relieve submarino

El suelo oceánico generalmente se presenta muy accidentado. Los sondeos revelan mesetas, cordilleras, colinas y profundas depresiones.

\section{Formas de relieve submarino:}

$>$ La plataforma continental.

$>$ Talud continental

$>$ Región pelágica

$>$ Región abisal

La plataforma continental: Es la parte del continente cubierto por las aguas del mar hasta una profundidad de 200 metros, donde se cree, penetran los rayos solares. La luz solar y las sustancias nutritivas que proceden de los continentes, contribuyen al desarrollo de la vida vegetal y animal; por eso en estas plataformas abundan peces, petróleo, minerales etc., y es donde se encuentran las áreas pesqueras mayores del mundo.

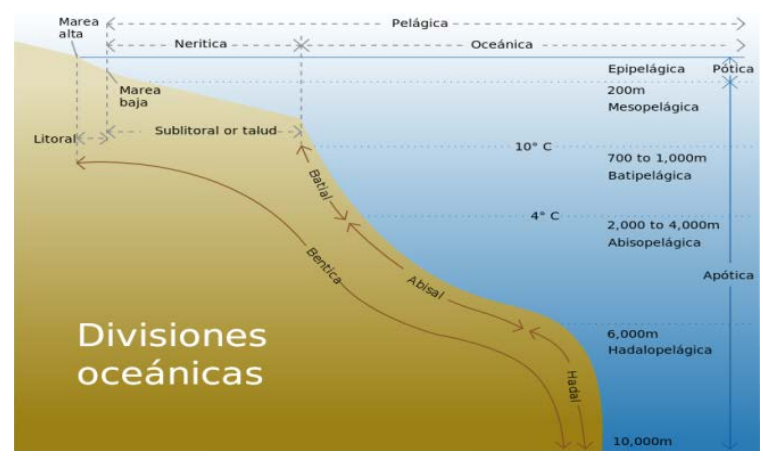

Figura 2.Relieve submarine (Fuente: Oceanic_divisions.svg: Chris huh)

\section{Energía oceánica}

Los océanos cubren más del 70\% de la tierra, actúan en el mundo como sistemas colectores de energía y que puede ser almacenada como energía térmica o energía cinética y también en un porcentaje menor como energía química y de productos biológicos. ${ }^{7}$

\section{Formas de energía de los océanos}

$>$ Energía de las corrientes.- Es la energía obtenida de las corrientes oceánicas o el aprovechamiento de la energía cinética de las corrientes marinas.

$>$ Energía Azul o potencia ósmica.- Es la energía de los gradientes de salinidad u obtenida por la diferencia de concentración salina entre el agua de mar y el agua de los ríos.

> Energía térmica oceánica.- Es la energía de las diferencias de temperatura a diferentes profundidades o entre la superficie y el fondo marino.

$>$ Energía Mareomotriz.- Energía que se obtiene mediante el aprovechamiento de las mareas.

> Energía Undimotriz o d e las olas.- Es la energía que se obtiene del aprovechamiento de las olas en los mares y océanos.

\section{Energía undimotriz}

La capacidad energética de un frente de ola se mide en densidad de energía por metro de frente y su valor varia de unas localizaciones a otras y ese potencial depende de la altura de ola y se atenúa con la profundidad del agua como consecuencia del rozamiento de esta con el fondo. ${ }^{8}$

Esta fuente energética no está cuantificada con exactitud, oscilando la estimación entre los $2.000 \mathrm{Gw}$ y los $4.000 \mathrm{Gw}$, por lo que repartiendo estos valores entre los $336.000 \mathrm{Km}$ de costa existentes en el mundo se obtiene un valor medio de densidad energética de las olas por metro de costa de $8,0 \mathrm{Kw}$, aunque esta distribución dista mucho de ser igualitaria ya que hay zonas de costa como es el caso de Australia o Nueva Zelanda donde se puede alcanzar los $100 \mathrm{Kw} / \mathrm{m}$ frente a otros lugares donde solo hay $5 \mathrm{Kw} / \mathrm{m}$. En Europa este recurso se encuentra mayoritariamente en la Costa Atlántica y oscila entre los $30 \mathrm{Kw} / \mathrm{m}$ y los $75 \mathrm{Kw} / \mathrm{m}$ según cual sea la localización. 


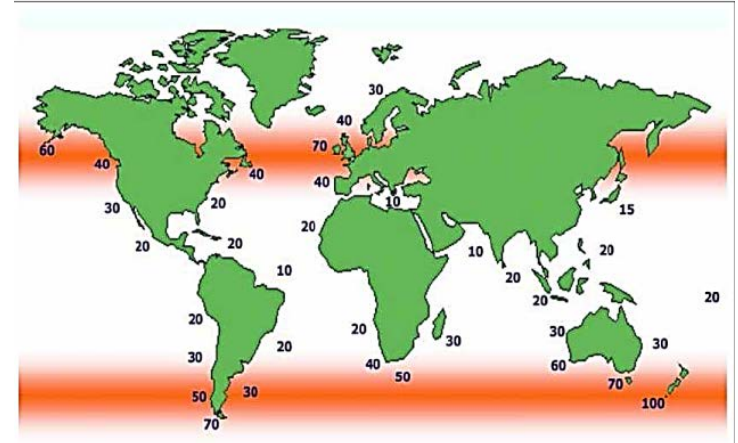

Figura 3. Niveles de Energía de las olas (kW/mfrente de ola).(Fuente: T.W. Thorpe "An Overview of Wave Energy Technology: Status, performance and Cost)

Potencial de la energía de los océanos

Tabla 1. Recursos energéticos marinos globales teóricos

\begin{tabular}{|c|c|c|}
\hline $\begin{array}{c}\text { Capacidad } \\
\text { (GW) }\end{array}$ & $\begin{array}{c}\text { Generación anual } \\
\text { (TWh) }\end{array}$ & $\begin{array}{c}\text { Forma de la } \\
\text { energía }\end{array}$ \\
\hline 5.000 & $\mathbf{5 0 . 0 0 0}$ & $\begin{array}{c}\text { corrientes } \\
\text { marinas }\end{array}$ \\
\hline 90 & 800 & mareas \\
\hline $2.000-4.000$ & $20.000-40.000$ & olas \\
\hline
\end{tabular}

Fuente: WEC (World Energy Council) Consejo Mundial De Energía

\section{Movimientos de las aguas oceánicas}

\section{Las olas.}

Son las oscilaciones de las partículas de agua, causadas por la fricción del viento sobre ella. Su tamaño es determinado por la velocidad y duración del viento.

\section{Tipos de olas}

Olas de oscilación: Se representan en toda la superficie del mar y se deben a las variaciones del nivel mar. En ellas el agua no avanza, solo describe un giro al subir y bajar casi en el mismo sitio en el cual se originó el ascenso de la ola.

Olas forzadas: Se producen por el viento y en ocasiones pueden ser altas como consecuencia de los huracanes.

Las olas libres: Que se forman en alta mar sin la presencia del viento, donde puede observarse que las olas "ruedan" por varios kilómetros.

Olas de traslación: Están cercanas a la costa, tocan el fondo, avanzan y se estrellan contra el litoral formando abundante espuma. $\mathrm{Al}$ regresar el agua hacia el mar se origina la resaca.

Tsunamis: Son ondas producidas por el efecto de un maremoto, o por una explosión volcánica, donde pueden ocurrir dos situaciones, la primera dondelas aguasse hunden enel centro de la perturbación, y segunda donde estas se levantan explosivamente.

\section{Parámetros de una ola}

El modelo de la figura $4, \eta(x, t)$ representa la elevación de la ola en función del tiempo y la distancia. En este modelo el parámetro $A$ representa la elevación máxima, wla velocidad angular temporal, $k$ la velocidad angular espacial, Tel tiempo, $x$ la distancia, $L$ la longitud, $H$ la altura, $h$ la profundidad, $C$ la velocidad del frente de ola 0 velocidad de fase y $S W L$ el nivel de la superficie marina.

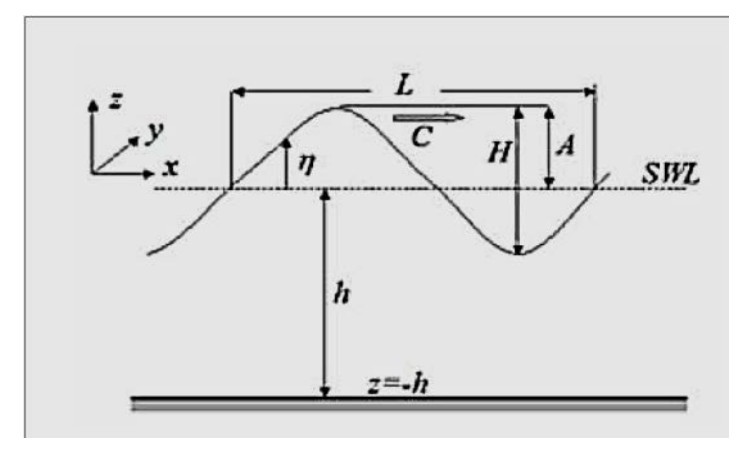

Figura 4. Parámetros de una ola (Fuente: Falnes 2004)

La energía de las olas: flujo de energía 9 .

$$
\begin{aligned}
& E_{\text {total }}=\frac{\rho * g * H^{2}}{8}=\frac{\rho * g * A^{2}}{2} \\
& F_{\text {energia }}=\frac{\rho * g * H^{2}}{8} * C_{g}=\frac{\rho * g * A^{2}}{2} * C_{g} \\
& F_{\text {energia }}=\frac{\rho * g^{2} * H^{2} * T}{32 \pi}=\frac{\rho * g^{2} * A^{2} * T}{8 \pi}
\end{aligned}
$$

En donde(Falnes 2004):

$\mathrm{E}_{\text {total }}=$ Energia total en

$\mathrm{F}_{\text {energia }}=$ Flujo de energia en $\mathrm{W} / \mathrm{m}$

$\rho=$ Densidad del agua en $1020 \mathrm{Kg} / \mathrm{m}^{3}$

$\mathrm{g}=$ Gravedad terrrestre $=9.81 \mathrm{~m} / \mathrm{seg}^{2}$

$\mathrm{H}=$ Altura de la ola en metros

$\mathrm{A}=$ Amplitud de la ola en metros $=\mathrm{H} / 2$

$\mathrm{C}_{\mathrm{g}}=$ Celeridad del grupo en $\mathrm{m} / \mathrm{seg}=\mathrm{C} / 2$

$\mathrm{C}=$ Celeridad de la onda $=\mathrm{L} / \mathrm{T}$

$\mathrm{L}=$ Longitud de la onda en metros $\rightarrow\left(\mathrm{g} * \mathrm{~T}^{2}\right) / 2 \pi$ (para profundidas indefinidas)

$\mathrm{T}=$ Periodo en segundos 
$\mathrm{k}=$ velocidad angular espacial $=2 \pi / \mathrm{L}$

Sistemas que convierten la energía undimotriz en energía eléctrica:

1. Empuje de la ola.

2. La variación de la altura en la superficie.

3. La variación de la presión bajo la superficie.

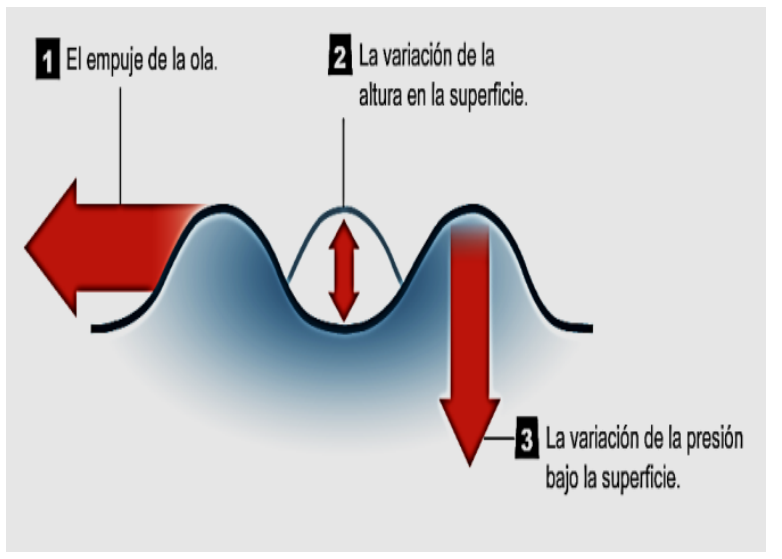

Figura 5. Sistemas que convierten la energía undimotriz en energía eléctrica.(Fuente: Centre 2004)

\section{Requerimientos para la instalación}

Aun cuando el trabajo y estudio realizado alrededor de este tipo de energía renovable es bastante bajo con relación a otras energías renovables, aparte de los costos de inversión necesarios para la implementación de los equipos y herramientas que permitan el correcto funcionamiento para obtener energía eléctrica a partir del movimiento de las olas, es necesario tener una serie de condiciones geológicas para su optimo uso:

\section{Profundidad}

Según estudios realizados a lo largo de la historia con respecto a esta energía renovable, se sabe que la cantidad de energía que se puede obtener a partir de ella, es proporcional al periodo de oscilación de las olas, al igual que al cuadrado de la amplitud de estas. Por tal razón se sabe que este tipo de características se hallan en territorios marítimos con profundidades entre 40 y 100 metros. Entre dichas profundidades las características de las olas resultan ser óptimas para la energía undimotriz.

La siguiente Tabla 2 muestra los valores de energía procedentes de este tipo de fuente de a la ubicación en el mar y su cercanía a la costa.
Tabla 2. Valores de energía en $K W / m$ según la profundidad donde se coloque el dispositivo

\begin{tabular}{|c|c|}
\hline Zona & Valores en KW/m \\
\hline Agua profundas & 70 \\
\hline Zonas cercanas a orilla & $20-30$ \\
\hline Orilla & 10 \\
\hline
\end{tabular}

Equipos $^{10,11}$

Los equipos de mayor uso en la actualidad para la implementación de este tipo de energía pueden clasificarse en dos grupos: fijos y flotanteslos que a su vez pueden ser:

\section{$>$ Dispositivos de columna de agua oscilante}

Estos funcionan con una turbina de aire que se encuentra ensamblada a un generador. Esta estructura normalmente se encuentra ubicada en la costa. Su parte inferior se encuentra sumergida abriéndose hacia el oleaje, en su parte superior contiene una cámara de aire. El aire se desplaza por la columna de agua generando un movimiento en la turbina ubicada en la parte alta del dispositivo. Se utiliza energía neumática para generar energía mecánica.

\section{$>$ Dispositivos oscilantes}

Estos funcionan con un motor hidráulico, turbina hidráulica y un generador eléctrico lineal.

\section{$>$ Dispositivos Flotadores}

Estas boyas contienen un sistema hidráulico el cual acciona un generador gracias al constante movimiento del oleaje haciendo que se genere un movimiento relativo entre el mástil y el flotador donde luego la salida eléctrica se lleva hasta una subestación.

\section{$>$ Dispositivos de Rotación}

Este sistema está formado por un módulo que se encuentra anclado al fondo marino y mediante las oscilaciones se accionan unos pistones que logran una transformación hidroeléctrica. Están constituidos principalmente por una estructura articulada donde las conexiones de los nodos disponende un sistema hidráulico que actúa sobre un generador eléctrico. Esta tecnología ya se encuentra en etapa comercial donde 30 de estos aparatos podrían generar energía a 20.000 hogares.

\section{Sistemas de Traslación lineal}

Estos sistemas están constituidos por dos partes: Una se encuentra fija sobre el fondo marino, y la otra se 
mueve de manera vertical por la variación de presiones hidrostáticas bajo el agua por las olas.

\section{$>$ Sistemas Colectores de olas}

Aprovechando la energía potencial de las olas, estos aparatos reciben energía al mover unas turbinas hidráulicas. El depósito se encuentra ubicado un nivel encima del mar donde a través de una rampa ingresan las olas, donde a continuación pasan por unas turbinas ensambladas a unos generadores eléctricos.

\section{$>$ Sistema Arquímedes Wave Swing}

Consiste en un cilindro de un peso de 800 toneladas, fijado al fondo y actúa sumergido a una profundidad de 10 metros. La parte superior es móvil y se desplaza en un movimiento vertical de arriba hacia abajo impulsando aire que acciona el sistema neumático de generación.

\section{$>$ Powerbuoy, El Pistón Marino}

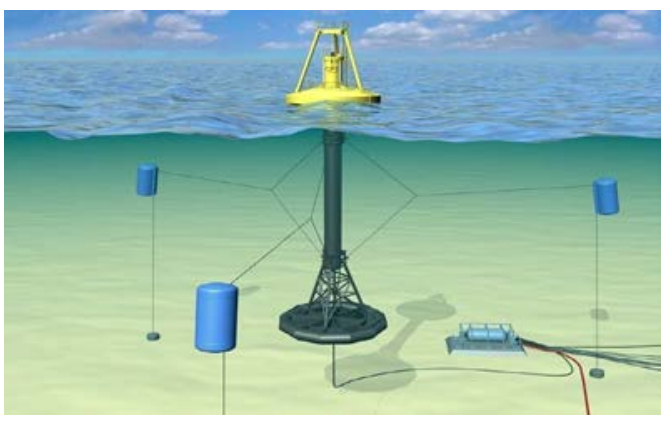

Figura 6. Boya de energía(Fuente:http://kresalaenergia.files.wordpress.com )

El PowerBuoy es un sistema con una estructura similar a un pistón que se mueve a medida que la boya sube y baja con las olas. El movimiento hace que un generador produzca electricidad. La mayor parte de la boya está sumergida en el agua, está diseñada para funcionar en profundidades de 30 a 50 metros.

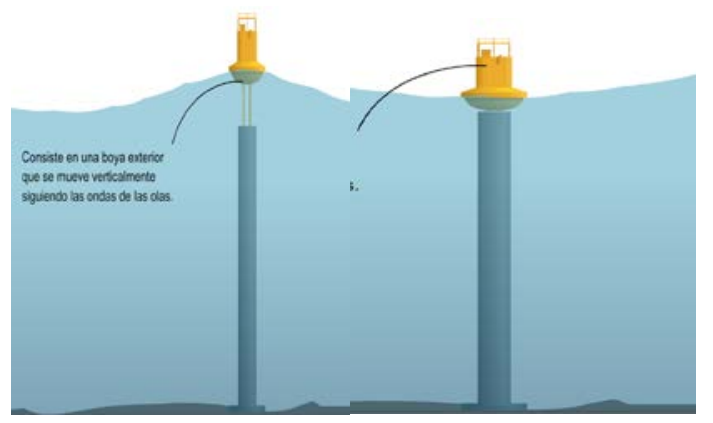

Figura 7. Boya de energía siguiendo el movimiento de las ondas de las olas(Fuente:ecomedioambiente.com)

ElPowerbuoy PB40 es un dispositivo que puede generar $40 \mathrm{KW}$ de potencia y una PB150 pues generara $150 \mathrm{KW}$ con un tope máximo de $866 \mathrm{KW}$ de potencia.

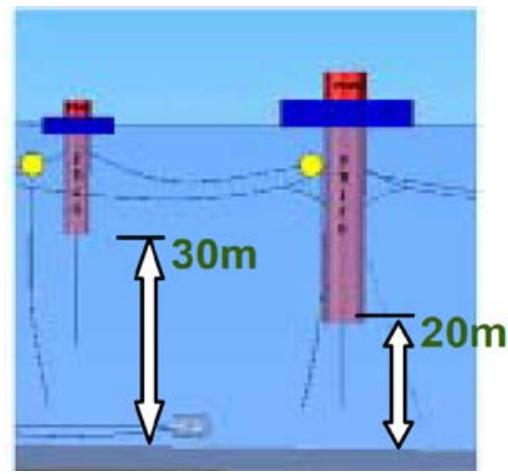

Figura 8. Powerbuoy PB40 “izquierda” y una Powerbuoy PB150

“derecha”(Fuente:http://www.oceanpowertechnologies.c om)

El sistema de generación de la onda dePowerBuoydel OPT (Ocean Power Technologies) utiliza una boya discretade alta mar para capturar y convertir energía de la onda en fuerza mecánica controlada que luego se colecta enun generador eléctrico.

El movimiento ascendente y descendente de las ondas de orilla hace que la boya se mueva libremente hacia arriba y hacia abajo. El rozamiento mecánico resultante que se produce en el interior de la boya activa el generador eléctrico. La corriente alterna generada se convierte en la corriente continua del alto voltaje que se transmite en tierra vía un cable de transmisión subacuático.

El PowerBuoy se realiza con sensores que supervisan continuamente el funcionamiento de variossubsistemas y del ambiente circundante del océano. Cuando se acercan olas demasiado grandes, el sistema se desconecta automáticamente. Cuando las alturas de las olas vuelven a lo normal, el sistema se reconecta y comienza la conversión y la transmisión de la energía.

\section{Requisitos de la tecnología del OPT
PowerBuoy ${ }^{13,14}$ :}

* Es construido de boyas resistentes con amarraduras convencionales probadas, anclando un cable subacuático en la transmisión. Evita cualquier daño o amenaza para el suelo del mar o la vida del mar. 
* Requiere solamente mantenimiento a un costo regular, con un periodo de vida útil de 30 años.

* Requiere instalación simple.

* El costo es altamente competitivo frente a otras fuentes convencionales de energía

* Es comparable a las centrales eléctricas de la alta capacidad (100MW+).

* La energía puede ser vertida inmediatamente en la red de energía o ser almacenada.

* Únicamente serán visibles los mástiles que se levantan sobre la superficie del agua, que pueden servir como ayudapara la navegación, por ejemplo un reflector del radar, una marca del día, y un piloto para ayudar a marineros a su localización.

* La presencia de las boyas no causa ninguna restricción significativa a la pesca. De hecho, las boyas sirven como filón artificial y atraen a los peces y otros tipos de vidas marinas. En algunas partes del mundo, las boyas convencionales se despliegan para servir como "dispositivos que sirven para atraer peces”.

* Aunque las boyas generan electricidad, todos los dispositivos de generación de transmisión eléctricos se contienen en recintos aislados y un cable subacuático estándar se blinda para prevenir corrientes eléctricas perdidas. El sistema se diseña para prevenir cualquier pérdida de electricidad o de emisiones de campos electromagnéticos.

* La energía es llevada a la orilla por un cable submarino estándar de pequeño diámetro de transmisión.

\section{Situación energética en el Ecuador.}

En el Ecuador existe diversos tipos de energías utilizadas para cubrir el sector eléctrico del país así se cuenta con aquellas provenientes de la energía hidráulica, turbo vapor, eólica y solar; siendo la hidráulica la que mayor aporte da al sistema eléctrico nacional, como también la energía térmica proveniente de combustibles fósiles.

Potencia instalada en el año 2012 comparada con años anteriores.

Las gráficas 1, 2, 3, muestran que en el año 2012 tenemos una variación en la potencia efectiva con respecto al año anterior de 5,79 \% y si comparamos con el 2002 se observa un aumento del 55,42 \%, obteniendo un aumento significativo pasando de 3293,19 MW en el 2002 a 5118,24 en el 2012. Solo tomando en cuenta la potencia generada en el Ecuador más no la importada o de interconexión.
Tabla 3. Potencia por tipo de energía y tipo de central

\begin{tabular}{|c|c|c|c|}
\hline \multirow[t]{2}{*}{$\begin{array}{l}\text { Tipo de } \\
\text { energía }\end{array}$} & \multirow[t]{2}{*}{ Tipo de central } & $\begin{array}{c}\text { Potencia } \\
\text { efectiva } \\
2011 \\
\end{array}$ & $\begin{array}{c}\text { Potencia } \\
\text { efectiva } \\
2012 \\
\end{array}$ \\
\hline & & MW & MW \\
\hline \multirow{4}{*}{ Renovable } & Hidráulica & 2207,17 & 2245,62 \\
\hline & Turbovapor & 93,4 & 93,4 \\
\hline & Eólica & 2,4 & 2,4 \\
\hline & Solar & 0,04 & 0,078 \\
\hline \multicolumn{2}{|c|}{ total de energía renovable } & 2303,01 & 2341,5 \\
\hline \multirow{3}{*}{ No Renovable } & térmica MCI & 1183,65 & 1348,6 \\
\hline & $\begin{array}{l}\text { térmica } \\
\text { Turbogas }\end{array}$ & 897,5 & 973,9 \\
\hline & $\begin{array}{l}\text { térmica } \\
\text { Turbovapor }\end{array}$ & 454 & 454,24 \\
\hline \multicolumn{2}{|c|}{ total de energía renovable } & 2535,15 & 2776,74 \\
\hline \multicolumn{2}{|c|}{ total } & 4838.16 & 5118,24 \\
\hline
\end{tabular}

Fuente: Boletín estadístico sector eléctrico ecuatoriano año 2011, CONELEC ${ }^{14}$

Los datos de la tabla 3 nos muestran que en la provincia de Santa Elena solo se genera electricidad a partir de térmicas con motores de combustión interna (MCI), y que el consumo en el año 2011, fue de 480,05 kwh de energía bruta, mientras que de potencia efectiva y potencia nominal se generó 130,1 MW.

Tabla 4.Potenciade la interconexión eléctrica

\begin{tabular}{|r|l|r|r|}
\hline \multirow{2}{*}{$\begin{array}{c}\text { Inter- } \\
\text { conexiones }\end{array}$} & Colombia & 525 & 525 \\
\cline { 2 - 4 } & Perú & 110 & 110 \\
\hline \multirow{2}{*}{ total de interconexión } & $\mathbf{6 3 5}$ & $\mathbf{6 3 5}$ \\
\hline \multicolumn{2}{|r|}{ total } & $\mathbf{5 4 7 3 , 1 6}$ & $\mathbf{5 7 5 3 , 2 4}$ \\
\hline
\end{tabular}

Fuente: Boletín estadístico sector eléctrico ecuatoriano año 2011, CONELEC 

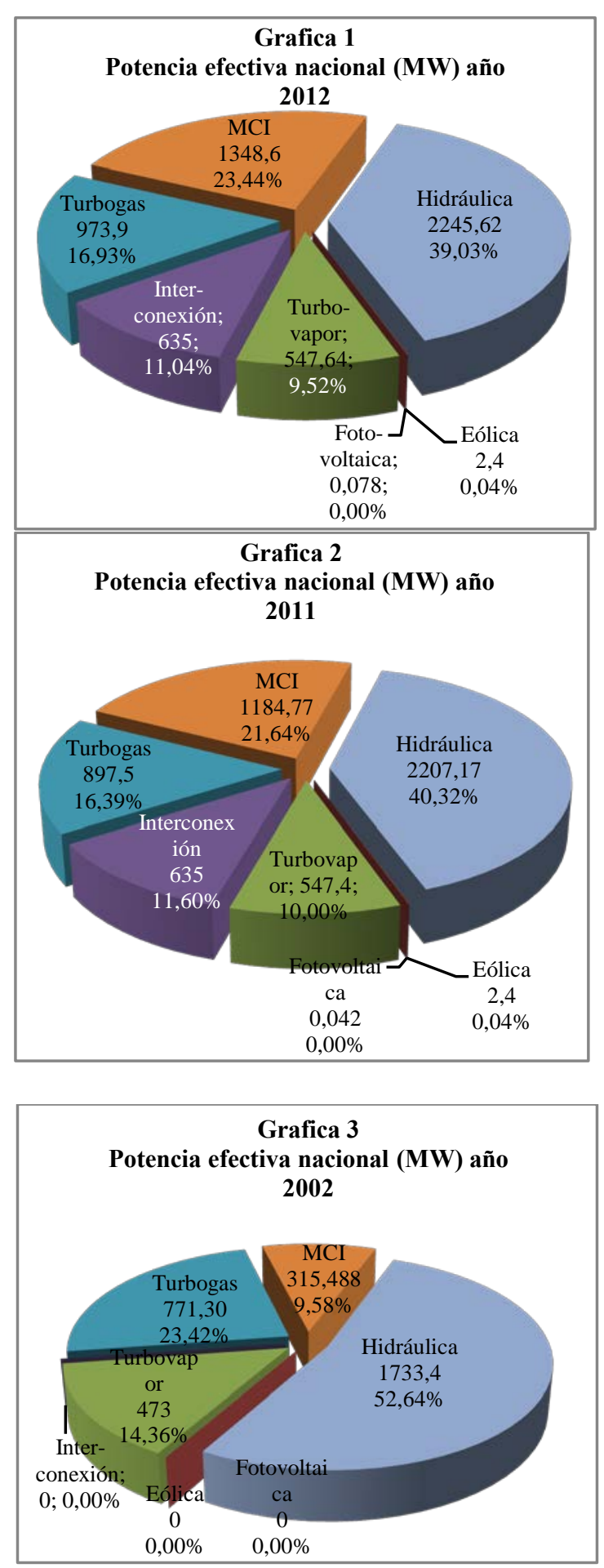

Fuente: CONELEC

Las gráficas 4, 5, 6, nos muestran que la potencia efectiva total del año 2012 que es de 5753,24 MW, de las cuales2776,74 MW es decir un48,26 \% corresponden a la generación de electricidad en centrales de energía no renovables, por otro lado2341,5 MW es decir un 40,70\% se debe a la generación en centrales de energía renovable, mientras que $635 \mathrm{MW}$ es decir el $11.04 \%$ corresponde a la interconexión que existe con Colombia y Perú.

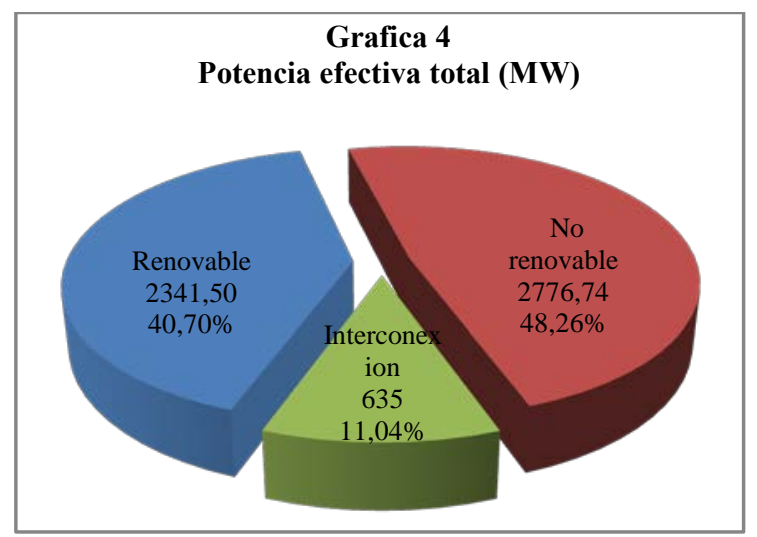

La hidráulica es la que más aporta a la producción en las energías renovables con un $95,91 \%$ es decir 2245,62 MW mientras que las térmicas MCI son las que más aportan en las energías no renovables con un 48,57\% es decir 1348,6 MW.
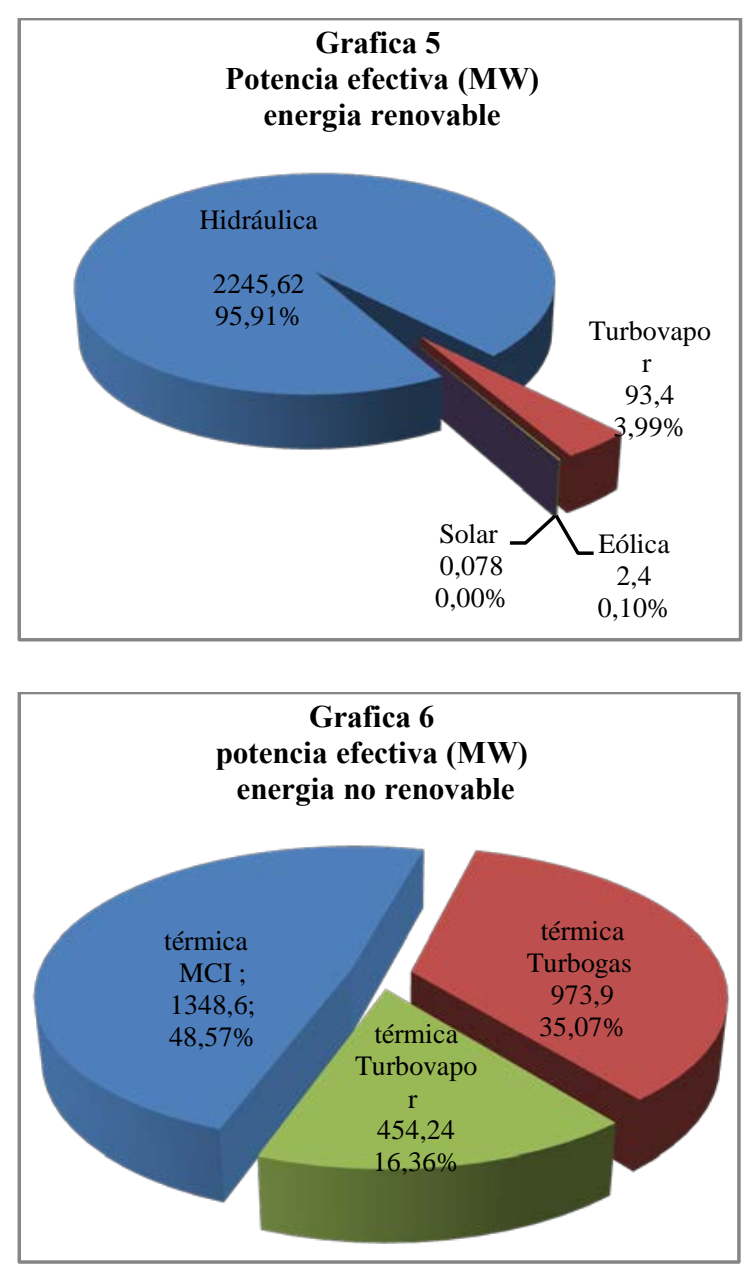

Fuente: CONELEC 
De acuerdo al contenido de la tabla 5del total de la energía bruta a nivel nacional en el 2012, el 54,3 \% corresponde a la energía producida por fuentes renovables, el 44,66 \% a la energía de fuentes no renovables y el 1,03 \% a la importación de energía. El mayor porcentaje de producción de energía por medio de fuentes renovables fue consecuencia de las mejores condiciones hidrológicas presentadas en las cuencas que alimentan las principales centrales hidroeléctricas.

Con un aumento de energía en comparación con el año anterior del 5,71\% es decir de 21838,73 GWh en el 2011 a 23085,92 GWh en el 2012.

Tabla 5 Producción bruta por tipo de energía y de central

\begin{tabular}{|c|c|c|c|}
\hline \multirow{2}{*}{$\begin{array}{l}\text { Tipo de } \\
\text { energía }\end{array}$} & \multirow{2}{*}{ Tipo de central } & \multicolumn{2}{|c|}{ Energía } \\
\hline & & GWh & $\%$ \\
\hline \multirow{4}{*}{ Renovable } & Hidráulica & 12237,72 & 53,01 \\
\hline & Turbovapor & 296,35 & 1,28 \\
\hline & Eólica & 2,4 & 0,01 \\
\hline & Solar & 0,33 & 0,00 \\
\hline \multicolumn{2}{|c|}{ total de energía renovable } & 12536,8 & 54,30 \\
\hline No renovable & térmicas & 10310,92 & 44,66 \\
\hline \multicolumn{2}{|c|}{ total de energía no renovable } & 10310,92 & 44,66 \\
\hline interconexión & interconexión & 238,2 & 1,03 \\
\hline \multicolumn{2}{|c|}{ total de interconexión } & 238,2 & 1,03 \\
\hline \multicolumn{2}{|c|}{ total } & 23085,92 & 100 \\
\hline
\end{tabular}

Fuente: CONELEC

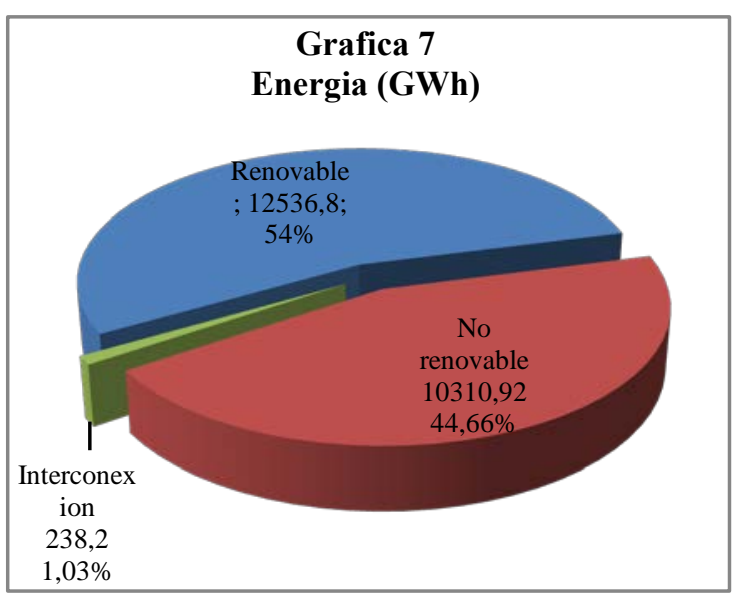

Grafico 7. Estructura de la producción bruta por tipo de energía.

Fuente: CONELEC
Tabla 6. Energía bruta, potencia nominal y potencia efectiva de las generadoras.

\begin{tabular}{|c|c|c|c|}
\hline Empresa & $\begin{array}{c}\text { CELEC - } \\
\text { Electroguayas }\end{array}$ & $\begin{array}{c}\text { CELEC- } \\
\text { termopichincha }\end{array}$ & TOTAL \\
\hline Central & Santa Elena II & Santa Elena & - \\
\hline Unidad & Santa Elena & Santa Elena & - \\
\hline $\begin{array}{c}\text { Tipo de } \\
\text { generación }\end{array}$ & térmica MCI & térmica MCI & - \\
\hline $\begin{array}{c}\text { Energía } \\
\text { bruta } \\
\text { (GWh) }\end{array}$ & 383,83 & 96,22 & 480,05 \\
\hline $\begin{array}{c}\text { Potencia } \\
\text { efectiva } \\
\text { (MW) }\end{array}$ & 90,1 & 40 & 130,1 \\
\hline $\begin{array}{c}\text { Potencia } \\
\text { nominal } \\
\text { (MW) }\end{array}$ & 90,1 & 40 & 130,1 \\
\hline
\end{tabular}

Fuente: Boletín estadístico sector eléctrico ecuatoriano año 2011, CONELEC

Características de las olas frente a la costa de la provincia de Santa Elena

La provincia de Santa Elena posee una zona costera que evidenciagrandes posibilidades para una posible extracción de este tipo de energía, no obstante valdría mencionar que las condiciones de olas es decir los parámetros de las mismas van desde la altura, la longitud y el periodo, donde una distancia de la costa ecuatoriana adecuada para una profundidad de 40 a 100 metros, y en donde la densidad de energía es $\mathrm{KW} / \mathrm{m}$ de frente de ola sea la másadecuada, se podría proponer una instalación de 15 Boyas De Energía (Powerbuoy) de la Ocean Power Technology “O.P.T", de $866 \mathrm{KW}$ cada una,con una potencia de generación total de 12,99 MW. Así se abastecería un 9,98 \% la demanda de potencia en la provincia de Santa Elena.

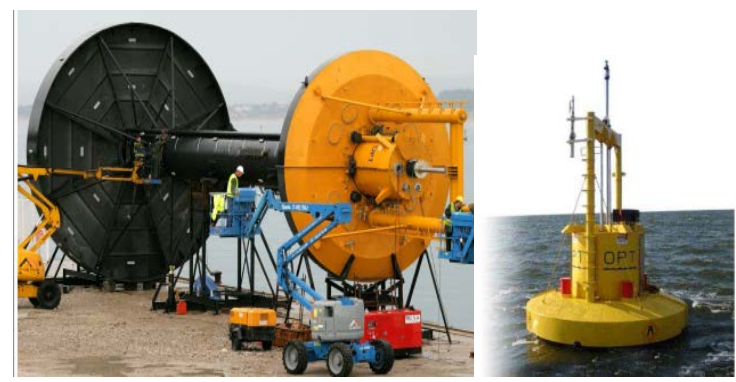

Figura 9.La boya Powerbuoy antes de ser instalada "izquierda" y ya instalada “derecha”(fuente:OceanPower Technologies) 


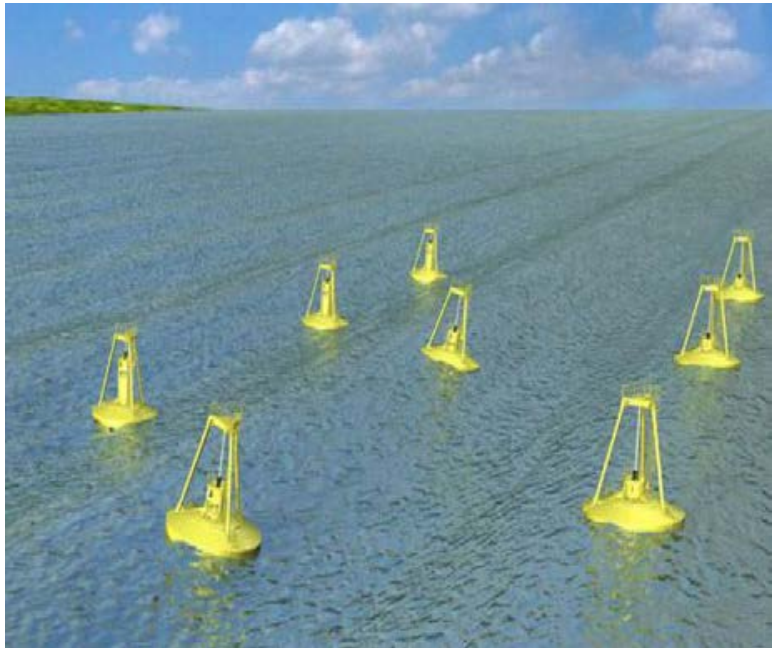

Figura 10.Central de boyas de energías (Fuente:http://www.mundo-geo.es/green-living)

\section{Ventajas y desventajas de la energía undimotriz}

\section{Ventajas:}

> La energía de las olas es gratuita. No es necesario algún tipo de combustible, y no produce residuos.

$>\quad$ No es caro de operar y obtener energía.

$>$ Puede producir una gran cantidad de energía.

$>$ Mantenimiento escaso.

$>$ Poco o ningún impacto ambiental.

\section{Desventajas:}

> Uno de los problemas técnicos importantes consiste en cómo absorber la energía mecánica, que incide en un campo aleatorio de velocidades, en energía eléctrica apta para su conexión a la red eléctrica.

$>$ El alto costo económico de la inversión inicial demanda que el periodo de amortización de estas centrales sea largo.

$>$ Su utilización se circunscribe a zonas costeras o próximas a la costa, por mayor erogación económica que implicaría transportar la energía obtenida a lugares del interior.

$>$ Otro inconveniente es el impacto ambiental debido a las instalaciones, que requieren modificación del paisaje para su construcción. Se ha de disponer de mucho espacio para albergar enormes turbinas, lo cual involucra un impacto ecológico sobre los ecosistemas, habitualmente costeros.
$>$ Por su carácter aleatorio, la cantidad de energía obtenida dependerá de los parámetros de la ola.

$>$ Es necesario un lugar adecuado para utilizar la energía del oleaje, donde las olas son generalmente fuerte.

\section{Conclusiones}

Los datos estadísticos de la generación eléctrica ecuatoriana, nos muestran que se sigue dependiendo en gran medida de los combustibles fósiles para la producción de electricidad, continuando con la contaminación de nuestro ambiente, y también se puede observar que hay falta de producción de electricidad obtenida de energías renovablesno convencionales, como la energía geotérmica, eólica, energías provenientes del mar (corrientes marinas, la maremotérmica, y la undimotriz), con lo cual se evitaría contaminar aún más muestro planeta, puesto que estas energías se caracterizan por el hecho de no contaminar y renovarse naturalmente o ser ilimitadas.

La energía undimotriz aún está en etapa de desarrollo tecnológico, por lo que provoca altos costos iniciales para su implementación. Pero ciertamente los avances tecnológicos en la actualidad muestran que este tipo de energía tiene un gran futuro, puesto que ya se han realizados instalaciones de dispositivos en escala real en varios países del mundo con resultados prometedores y alentadores, los cuales permiten mencionar que el Ecuador por ser un país costero, podría tener un gran potencial para instalaciones de dispositivos futuros para la obtención de energía eléctrica a partir de la energía de las olas.

El Inocar (Instituto Oceanográfico de la Armada Ecuatoriana) ha realizado mediciones de olas en sitios específicos de la zona costera ecuatoriana, instalando equipos oceanográficos entre los 10 y 20 $\mathrm{m}$. de profundidad, lo que representa una distancia promedio de $2 \mathrm{~km}$ desde la línea de costa hasta el punto de medición.

Las mediciones se han realizado por cortos períodos, es decir durante pocos meses, en la mayoría de casos, lapso que no cubre toda la variabilidad estacional o interanual y que por lo tanto no garantizaría que la altura encontrada sea representativa de la altura del sector.

Para la obtención de datos de olas para una cobertura espacial mayor, es decir a profundidades mayores y con mayor densidad de datos por área, se aplica modelamiento numérico, proceso que actualmente se encuentra en estudio $y$ en fase preliminar. Necesitándose posteriormente estudios de validación con mediciones in situ y de satélites 
Una vez obtenida esta información se podría proponer instalaciones con potencial de generación no tan altos debido a que las tecnologías actuales se encuentran en etapa de desarrollo, inclusive en el Reino Unido se han propuesto instalar plantas de generación undimotriz de solo $10 \mathrm{MW}$ de potencia total, con lo que se da a entender que inclusive en lugares en donde las tecnologías están más avanzadas, solo se plantea implementarlas en escalas menores.

Mayores estudios sobre las características de las olas nos podrían indicar la "posibilidad real” de explotar la energía undimotriz para la generación de energía eléctrica, frente a la costa de la provincia de Santa Elena.

\section{Referencias}

1. Falcão, A.F. de O. (2011). Wave energy utilization: A review of the technologies. Renewable and Sustainable Energy Reviews, 14, 899-918.

2. EREC. (2010). Renewable Energy in Europe, Markets, Trends and technologies. European Renewable Energy Council.

3. Polinder, H. \&Scuotto, M. (2005). Wave energy converters and their impact on power systems. International Conference on Future Power Systems. 62-70, Amsterdam.

4. Javier Martín Vide (2008) - La nueva realidad del calentamiento global: Un decálogo del cambio climático -Revista Electrónica de Geografía y Ciencias Sociales 270:23 01/08/2008 -Departamento de Geografía Física, Universidad de Barcelona, http://www.ub.edu/gc/Castella/Javier

Martin_Vide.htm.
5. Ley del régimen del sector eléctrico http://www.conelec.gob.ec/normativa_detalle.ph p?cd_norm=203.

6. The second International Symposium on Wave Energy Utilization, Trondheim, 22-24 June 1982.http://ntnu.divaportal.org/smash/get/diva2:550041 /FULLTEXT01.pdf.

7. Godfrey Boyle, Renewable Energy, $2^{\text {nd }}$ Edition, 2004, Oxford University Press.

8. S. H. Salter, Nature 249, 720 (1974).

9. Falnes J (2007). A review of wave-energy extraction, Marine Structures, 20, (4), 185-201.

10. J. Callaghan, R. Boud, Future Marine Energy Rep. CTC601, 2006.

11. J. Cruz, Ed. Ocean Wave Energy: Current Status and Future Perspectives (Springer, Berlin, 2008).

12. Ocean Power Technologies Inc. OPT (http://www.oceanpowertechnologies.com/).

13. Pelamis Wave Power Ltd. (http://www.pelamiswave.com/)

14. Boletín estadístico sector eléctrico ecuatoriano año 2011, CONELEC (Consejo Nacional De Electricidad) 
